\title{
Performance Analysis of Hybrid TB-LEACH Enegy based Multihop Protocol
}

\author{
Gaganpreet Kaur \\ Assistant Professor \\ Dept. of Computer \\ Engineering and Technology \\ Guru Nanak Dev University, \\ Amritsar, India
}

\author{
Gagandeep Kaur Virk \\ Assistant Professor \\ Dept. of Computer \\ Engineering and Technology \\ Guru Nanak Dev University, \\ Amritsar, India
}

\begin{abstract}
An energy efficient routing protocol named Hybrid TBLEACH Energy Based Multihop Protocol is proposed. The proposed protocol selects sensor nodes having higher residual energy as Cluster Heads which communicate with the base station by the means of minimum spanning tree. It has reduced energy consumed by sensor nodes. In this paper, performance of proposed protocol is analyzed with the effect of change in position of base station. The performance of proposed protocol is compared with the previous proposed protocols LEACH, TB-LEACH and CTPEDCA protocol.
\end{abstract}

\section{Keywords}

LEACH; Wireless Sensor Network; TB-LEACH; CTPEDCA

\section{INTRODUCTION}

Wireless sensor networks are made up of hundreds or thousands of sensor nodes which operates in an unattended environment for monitoring of the physical or environmental conditions. These nodes pass on (deliver) the sensed data to BS either directly or by the means of other sensor nodes. They communicate via radio links $[2,4]$

Two main challenges in a sensor network are savings of energy and extension of the lifetime of the network. Large amount of energy is consumed by nodes during communications. For energy-efficient communications among the nodes large number of routing protocols has been proposed. Routing protocols achieve this by selecting minimal routes. Routing protocols which work on the principle of clustering are best known for fulfilling main constraints of the WSNs such as reducing energy consumption, extending network lifetime and stability period of the network. $[1,3]$

Due to constraints on the size of sensor nodes, these cannot be outfitted with ample energy supplies so energy is provided by using small batteries. As the nodes are deployed in harsh environment so recharging or replacement of battery is not possible in most of the applications. So, many energy optimization methods have been proposed for sensor networks.

Large number of sensor nodes are disseminated in a wireless sensor network so data which is collected from sensors is highly redundant. This property of the nodes can be used as a benefit for extending life time of the network. Data aggregation is performed which reduce the amount of data transmitted to the sink. During data aggregation the data is collected from multiple sensors at intermediate nodes and aggregated data is communicated to the BS. Data aggregation reduces energy consumption by miniaturizing the number of transmissions. [4, 5]

Many variants of LEACH protocol have been proposed. By considering LEACH protocol as standard routing protocol of Wireless Sensor Network, our proposal protocol works on the framework of LEACH. Proposed protocol is combination of the two extensions of LEACH protocol named TB-LEACH and CTPEDCA (A cluster-based and tree-based power efficient data collection and aggregation protocol for wireless sensor networks) and two additional factors residual energy of nodes and distance of cluster head nodes from the base station are also considered.

\section{RELATED WORK}

In [7] LEACH has been proposed B. Heinzelman et. al. LEACH achievse the goal of distribtion of energy load by rotating the cluster head role among the sensor nodes. Each round of LEACH protocol works in two phases: steady phase proceededby setup phase.

In setup phase clusters are organized. In this phase nodes formulate a decision regarding acting as a cluster-head or as a normal node for the current round. Nodes makes this decision by selecting a number from 0 to1. If the selected number is lower than threshold then node become cluster head for that round otherwise it act as a normal node. The fomula used for calculating threshold is given as

$$
T(n)= \begin{cases}\frac{P}{1-P\left(r \bmod \frac{1}{P}\right)} & \text { if } n \in G \\ 0 \quad & \text { otherwise }\end{cases}
$$

Where $\mathrm{P}$ is a parameter which is equal to the ratio of number of desired $\mathrm{CHs}$ and total number of nodes $\mathrm{n} . \mathrm{r}$ is the round number and $\mathrm{G}$ is the set of nodes which have not taken the role of $\mathrm{CHs}$ in last $\mathbf{1 / P}$ rounds. The other nodes which are not chosen as cluster heads join the cluster heads and clusters are formed.

In Steady State phase nodes chosen as cluster heads send data to base station after collecting data from the local cluster members.

In [1] DE-LEACH is proposed by Surender Kumar et. al. In this protocol different schemes are used for electing the cluster heads depending on whether nodes are near to base station or far from the BS. They have considered the results of LEACH protocol that system is more energy efficient when cluster heads are between $3 \%$ and $5 \%$. The sensing region is divided into two parts. First region contain all those nodes whose distance is less than or equal to average distance from base station, the cluster heads are elected on the basis of distance of nodes from the base station and percentage of 
cluster heads is slightly more than 6 percent. Second region contain all those nodes whose distances are more than the average distances from the base station, the cluster heads are selected on the basis of residual and initial energy of the sensor nodes and desired percentage of cluster heads is slightly more than 3 percent. These schemes increase the chances of nodes which are in the center of sensing region to become cluster head.

In [2] DSC protocol is proposed by Fuad Bajaber et. al. This protocol makes use of both static and dynamic clustering schemes. This protocol is extension of LEACH-C protocol. Dynamic clustering consists of two phases. In setup phase cluster heads are selected and clusters are organized by the base station. In steady phase sensor nodes transmits data and information of the energy status to the cluster heads. Cluster heads fuse this data and transmit it to the base station. Cluster heads choose new cluster heads for next round by taking into account energy status of sensor nodes. Principle of static clustering is used after this. Static clustering is used only in the steady state phase. Clusters once formed are fixed for 10 rounds. Only the cluster head role is rotated among the nodes of the cluster. If the round number is less than 10, cluster heads transmit data to the base station after receiving from cluster members and select the sensor nodes with more energy as new cluster heads for next round. If round number is equal to 10 , sensor nodes transmit their location information and energy status to base station and set up phase begins. This scheme has reduced the setup phase overhead.

In [7] RDCS protocol is proposed by Hui Gao et. al. This protocol has modified the threshold value to include the residual energy of sensor nodes and their relative distances from the base station. For calculating the relative distance at the beginning base station broadcasts a 'hello' message at a certain power level and this original power level is saved in the message. Then nodes estimate their relative distance to the base station by comparing received signal strength of the message with original power level. After selection of cluster heads clusters are formed. Steady state phase of this protocol is same as LEACH protocol.

In [8] EAC protocol is proposed by Messai MohamedLamine. This protocol operates in two phases: setup phase and steady state phase. In set up phase all the nodes broadcast their current energy level. Each node arranges energy values which they have received from their neighbors. The node which has highest energy elect itself as cluster head, other nodes send join request messages to the neighbor having highest residual energy. Clusters are formed. Cluster heads send the TDMA schedule to their cluster members. Steady state phase is same as LEACH protocol.

In [9] LEACH-CC protocol is proposed by Baiping Li et. al. In this protocol centralized algorithm is used for selecting cluster heads. At the starting of each round all the nodes send their location and energy information to the base station. Base station using selects $\mathrm{k}$ nodes as the cluster heads by using optimization algorithm and ensures that cluster heads are dispersed throughout the network. Cluster heads send their data to the base station using chain routing. Cluster head having highest residual energy is selected as the leader node in each round. Each cluster head transmit to and receive from the neighbor cluster head, only the leader node has to transmit to the base station. By allowing only node to communicate with base station this protocol has reduced the dissipation of energy and increased life time of the network.
In [13] TB-LEACH protocol is proposed by Hu Junping et. al. In this protocol in each round constant number of nodes are elected as cluster heads by using random timer. To ensure that always a constant number of cluster heads are chosen a counter is used. At the starting of each round every node generates a random timer and start expiring it. When the timer of a node got expired, no. of received CH_ADV messages are checked by the node, if it is lower than four then it declares its status as a cluster head and whole process after the selection of cluster heads is same as LEACH protocol.

In [10] CTPEDCA protocol is proposed by Wei Wang et. al. This protocol has combined spanning tree strategy and clustering. The cluster heads are selected by using same strategy as LEACH protocol. In each round once cluster are selected, they construct a spanning tree. Highest energy cluster head node is elected as root node of the spanning tree. The cluster heads deliver aggregated data along the tree and finally the root node delivers data to the Base station.

\section{HYBRID TB-LEACH ENERGY BASED MULTIHOP PROTOCOL}

This protocol works in rounds. Each round has four phases: Selection of Cluster heads, Formation of Clusters, Formation of minimum spanning Tree and Data transmission. [15] The flow chart of the proposed protocol is given below:

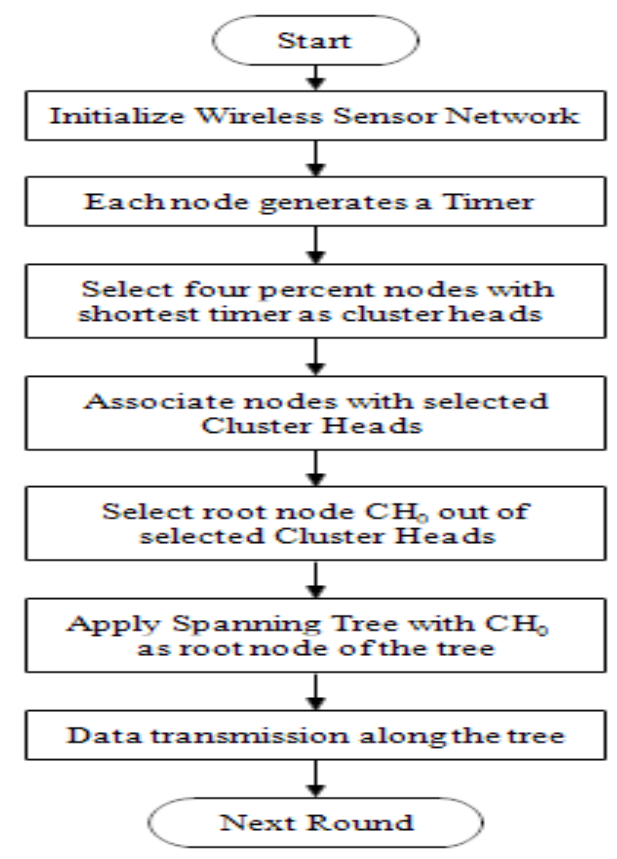

Fig 1: Flowchart of Proposed Protocol ${ }^{[15}$

The detail of each phase is given below.

\section{Phase 1: Selection of Cluster Heads}

In proposed protocol timer is assigned to all the nodes. The nodes having the smaller time become cluster heads. In each round time interval is chosen by nodes which depend on their residual energy. Initially all the nodes have same initial energy so for first round all the nodes choose the random timer and after first round the timer chosen by the nodes depend on their residual energy.

The timer chosen by nodes is given as 


$$
\operatorname{Timer}(\mathrm{i})=\left\{\begin{array}{lr}
\text { randi }(\operatorname{maxlimit}, 1,1) & \text { if } \mathrm{r}=0 \\
\frac{\mathrm{K}}{\text { residualenergy(i) }} & \text { otherwise }
\end{array}\right.
$$

Where $\mathrm{r}$ represents round number, $\mathrm{i}$ represents id of sensor node, and $\mathrm{K}$ is a positive integer, Initially $\mathrm{K}$ is given some highest value. Value of $\mathrm{K}$ is dynamically modified by the root node in phase 3 .

As the timer given to the sensor nodes depend on their residual energy, the nodes having higher residual energy acquire shortest timer and get more chance to become cluster head. When timer of any node expires it will check no. of advertisement messages received so far if it is less than the limit of the counter it will broadcast advertisement message using non persistent CSMA MAC protocol otherwise it cannot become cluster head for the current round. [15]

\section{Phase 2: Formation of Clusters}

In this clusters are formed using same criteria as LEACH protocol. [15]

\section{Phase 3: Formation of Minimum Spanning Tree}

After formation of clusters each node which is elected as cluster head broadcast the advertisement message containing information such as id of cluster head, id of cluster, information of location, cluster size, a parameter ED ( residual energy of cluster head divided by distance of cluster head node from the base station) [14]. Each cluster head node receives and save this information. Finally each cluster head get a table which contains information of all cluster heads. Then the cluster head having highest value of ED (residual energy divided by distance of cluster head node from the base station) parameter is selected as root node of the tree. Then the function for creating minimum spanning tree is called which is implemented by root node. After creation of minimum spaning tree the root node broadcasts the tree information message to other cluster heads. This message contain data transmission schedule among the cluster heads. The root node also modifies the value of $\mathrm{K}$ as:

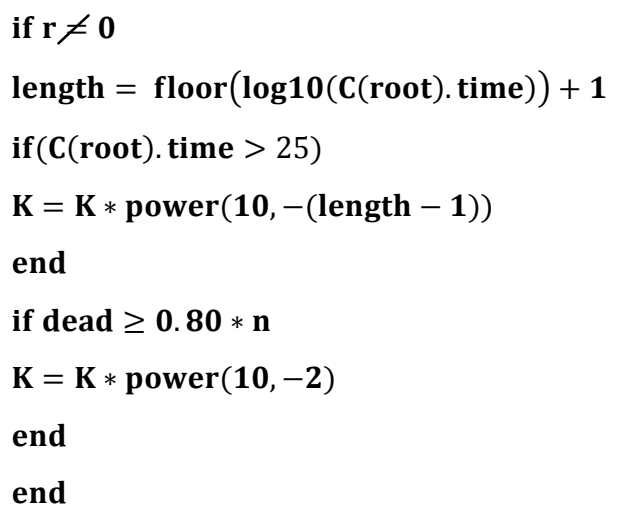

Where $\mathrm{r}$ represents round number, root represents the root node of the spanning tree, $\mathrm{n}$ is the total number of nodes in the network.

In each round except first round root node checks the two conditions.

Condition 1: Root node checks the time assigned to it during that round if it is greater than 25 then it finds the length of time allotted to it (no. of digits in the time allotted to it). It multiplies the value of $\mathrm{K}$ by $10^{- \text {(length-1) }}$.
Condition 2: Root node also checks for the no. of dead nodes. If $80 \%$ or above $80 \%$ of the nodes are dead then it multiplies the value of $\mathrm{K}$ by $10^{-2}$.

If any of the above condition is satisfied, root node modifies the value of $\mathrm{K}$. The value of the $\mathrm{K}$ is modified according to above these two conditions so that the waiting timer does not go very high.

Root node broadcasts this value to other cluster heads. After receiving from root node, cluster heads broadcast this value of $\mathrm{K}$ to their cluster members. [15]

\section{Phase 4: Data transmission}

In this phase non cluster head nodes send their data to their respective cluster head node during their allocated time slot. Once the cluster head nodes have received data from their cluster members, they aggregate this data and send along the tree. When all cluster heads have completed collection and aggregation of data from their local cluster nodes, they send their data by means of the spanning tree. Each receiving cluster head performs aggregation of data received from the previous cluster head in the tree and local cluster data and transmitting it to next cluster head. Final resultant data is delivered by root node to the base station. [15]

\section{SIMULATION ANALYSIS}

In order to analyze the effect with change in position of base station LEACH, TB-LEACH, CTPEDCA and proposed protocols are simulated using MATLAB. The performance is evaluated on the basis of Dead Node Analysis by considering metrics FND (round at which first node dies), HND (round at which half nodes die), and LND (round at which last node die). FND, HND and LND are defined below in detail.

FND: FND refers to the round number at which first node of the network runs out of energy. The period from the $1^{\text {st }}$ round to the round number at which first node dies is called stability period.[11, 12]

HND: HND refers to the round number at which half of the nodes of the network die. [11, 12]

$L N D$ : LND refers to the round number at which last node of the network dies. LND represents the network lifetime. The period between FND and LND is called instability period. $[10,11]$

\subsection{Network Model}

100 homogeneous nodes are deployed randomly in the network area of $100 \times 100 \mathrm{~m}^{2}$. The Base station is located outside the region. Base station and sensor nodes are immobile.

\subsection{Simulation Parameters}

The parameter values which are used for simulation are listed below in the Table I.

Table 1. Table of values of the parameters

\begin{tabular}{|c|c|}
\hline Parameters & Values \\
\hline Number of Sensors & 100 \\
\hline Dimensions of Network & $100 \mathrm{~m} \times 100 \mathrm{~m}$ \\
\hline Initial Energy & $0.5 \mathrm{~J}$ \\
\hline $\begin{array}{c}\text { Transmitter Electronics } \\
(\text { Eelec })\end{array}$ & $50 \mathrm{~nJ} / \mathrm{bit}$ \\
\hline
\end{tabular}




\begin{tabular}{|c|c|}
\hline Receiver Electronics(Eelec) & $50 \mathrm{~nJ} / \mathrm{bit}$ \\
\hline $\mathrm{E}_{\mathrm{fs}}$ & $10 \mathrm{pJ} / \mathrm{bit} / \mathrm{m}^{2}$ \\
\hline $\mathrm{E}_{\mathrm{mp}}$ & $0.0013 \mathrm{pJ} / \mathrm{bit} / \mathrm{m}^{4}$ \\
\hline $\mathrm{E}_{\mathrm{DA}}$ & $5 \mathrm{~nJ} / \mathrm{bit} / \mathrm{signal}$ \\
\hline $\mathrm{d}_{0}$ & $\mathrm{Sqrt}\left(\mathrm{E}_{\mathrm{fs}} / \mathrm{E}_{\mathrm{mp}}\right)$ \\
\hline Data Packet length & $4200 \mathrm{bits}$ \\
\hline $\mathrm{P}$ & 0.04 \\
\hline Initial Value of $\mathrm{K}$ & 1 \\
\hline & a. $(50,200)$ \\
Base Station Location & b. $(50,225)$ \\
& c. $(50,250)$ \\
& d. $(50,275)$ \\
& e. $(50,300)$ \\
\hline
\end{tabular}

\subsubsection{Dead Node Analysis}

4.3.1.1 When Base Station is located at $(50,200)$

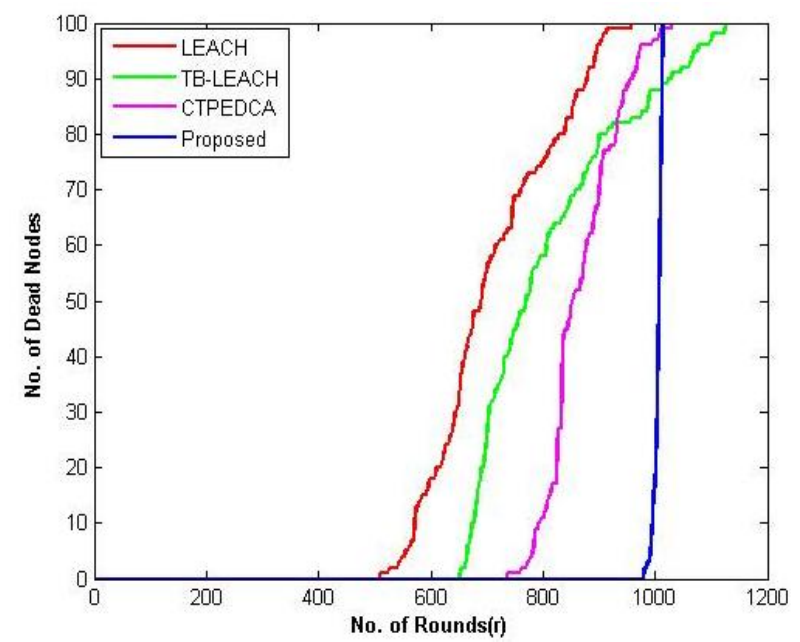

Fig 3: Number of nodes dead over Simulation time when base station is located at $(\mathbf{5 0 , 2 0 0 )}$

The above fig. 3 compares the number of dead nodes at each round in LEACH, TB-LEACH, CTPEDCA and proposed protocol. The values of FND, HND and LND of all the four protocols are given below in the table II.

Table 2. Fnd, Hnd And Lnd Comarison At (50,200)

\begin{tabular}{|l|l|l|l|l|}
\hline $\begin{array}{l}\text { Base } \\
\text { station } \\
\text { Loaction }\end{array}$ & Protocols & FND & HND & LND \\
\hline$(50,200)$ & LEACH & 508 & 691 & 957 \\
\cline { 2 - 5 } & TB-LEACH & 651 & 770 & 1125 \\
\cline { 2 - 5 } & CTPEDCA & 735 & 851 & 1029 \\
\cline { 2 - 5 } & Proposed & 980 & 1007 & 1013 \\
\hline
\end{tabular}

$F N D$ :It is clear from the table that first node dies later in the proposed protocol than the other three protocols. FND of the proposed protocol is approximately 1.9 times of LEACH, 1.5 times of TB-LEACH and 1.3 times of CTPEDCA.

Thus Stability period of proposed protocol is prolonger than LEACH, TB-LEACH and CTPEDCA protocols. CTPEDCA protocol is more stable than TB-LEACH and LEACH, while stability period of TB-LEACH is longer than LEACH protocol.

HND: HND of proposed protocol is approximately 1.4 times of LEACH protocol, 1.3 times of TB-LEACH and 1.2 times of CTPEDCA protocol. HND of CTPEDCA protocol is more than TB-LEACH and LEACH, while HND of TB-LEACH is more than LEACH protocol.

LND: The LND of TB-LEACH protocol is more than LEACH, CTPEDCA and Proposed protocol. 


\subsubsection{When Base station is located at $(50,225)$}

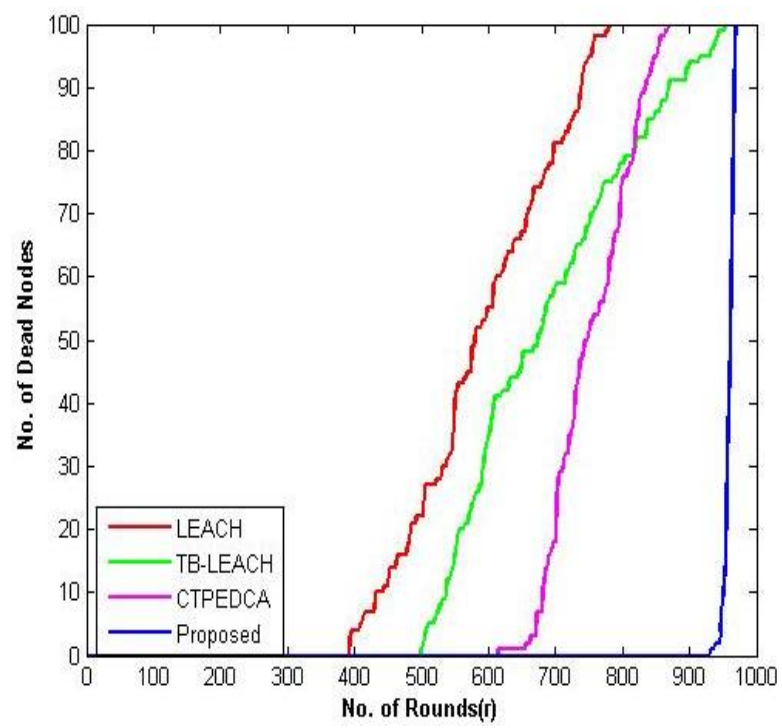

Fig 4: Number of nodes dead over Simulation time when base station is located at $(\mathbf{5 0 , 2 2 5 )}$

TABLE 3. FND, HND and LND comparison at $(\mathbf{5 0 , 2 2 5 )}$

\begin{tabular}{|l|l|l|l|l|}
\hline $\begin{array}{l}\text { Base station } \\
\text { Loaction }\end{array}$ & Protocols & FND & HND & LND \\
\hline \multirow{3}{*}{$50,225)$} & LEACH & 393 & 579 & 780 \\
\cline { 2 - 5 } & TB-LEACH & 498 & 673 & 953 \\
\cline { 2 - 5 } & CTPEDCA & 614 & 743 & 868 \\
\cline { 2 - 5 } & Proposed & 930 & 961 & 968 \\
\hline
\end{tabular}

FND: It is clear from the table that first node dies later in the proposed protocol than the other three protocols. FND of the proposed protocol is approximately 2.3 times of $\mathrm{LEACH}$, 1.9 times of TB-LEACH and 1.5 times of CTPEDCA.

Thus Stability period of proposed protocol is prolonger than LEACH, TB-LEACH and CTPEDCA protocols. CTPEDCA protocol is more stable than TB-LEACH and LEACH, while stability period of TB-LEACH is longer than LEACH protocol.

$H N D:$ HND of proposed protocol is approximately 1.6 times of LEACH protocol, 1.4 times of TB-LEACH and 1.3 times CTPEDCA protocol. HND of CTPEDCA protocol is more than TB-LEACH and LEACH, while HND of TB-LEACH is more than LEACH protocol.

The proposed protocol has improved the network life time about $24 \%$ as compared to LEACH, about $1 \%$ as compared to TB-LEACH and about $11 \%$ as compared to CTPEDCA. The CTPEDCA and TB-LEACH protocols have improved the network life time as compare to LEACH protocol. The instability period of proposed protocol is smallest as compared to other three protocols.

\subsubsection{When Base station is located at $(50,250)$}

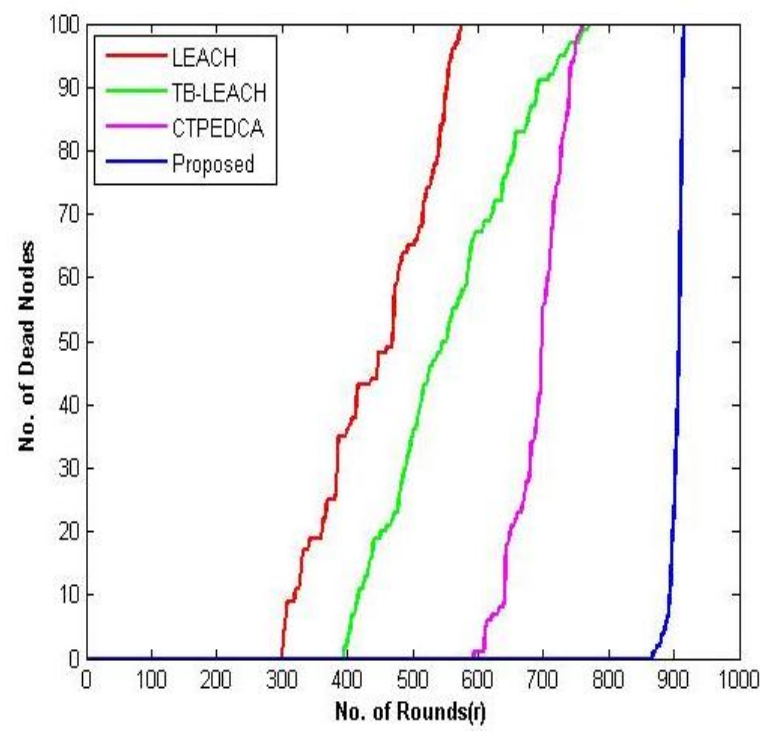

Fig. 5 Number of nodes dead over Simulation time when base station is located at $(\mathbf{5 0 , 2 5 0 )}$

Table 4. FND, HND and LND comparison at $(50,250)$

\begin{tabular}{|l|l|l|l|l|}
\hline $\begin{array}{l}\text { Base station } \\
\text { Loaction }\end{array}$ & Protocols & FND & HND & LND \\
\hline$(50,250)$ & LEACH & 301 & 469 & 574 \\
\cline { 2 - 5 } & TB-LEACH & 393 & 545 & 769 \\
\cline { 2 - 5 } & CTPEDCA & 593 & 698 & 759 \\
\cline { 2 - 5 } & Proposed & 867 & 907 & 914 \\
\hline
\end{tabular}

FND: It is clear from the table that first node dies later in the proposed protocol than the other three protocols. FND of the proposed protocol is approximately 2.8 times of LEACH, 2.2 times of TB-LEACH and 1.5 times of CTPEDCA.

Thus Stability period of proposed protocol is prolonger than LEACH, TB-LEACH and CTPEDCA protocols. CTPEDCA protocol is more stable than TB-LEACH and LEACH, while stability period of TB-LEACH is longer than LEACH protocol.

HND of proposed protocol is approximately 2.1 times of LEACH protocol, 1.6 times of TB-LEACH and 1.3 times CTPEDCA protocol. HND of CTPEDCA protocol is more than TB-LEACH and LEACH, while HND of TB-LEACH is more than LEACH protocol.

The proposed protocol has improved the network life time about $61 \%$ as compared to $\mathrm{LEACH}$, about $17 \%$ as compared to TB-LEACH and about $19 \%$ as compared to CTPEDCA. The CTPEDCA and TB-LEACH protocols have improved the network life time as compare to LEACH protocol. The instability period of proposed protocol is smallest as compared to other three protocols. 


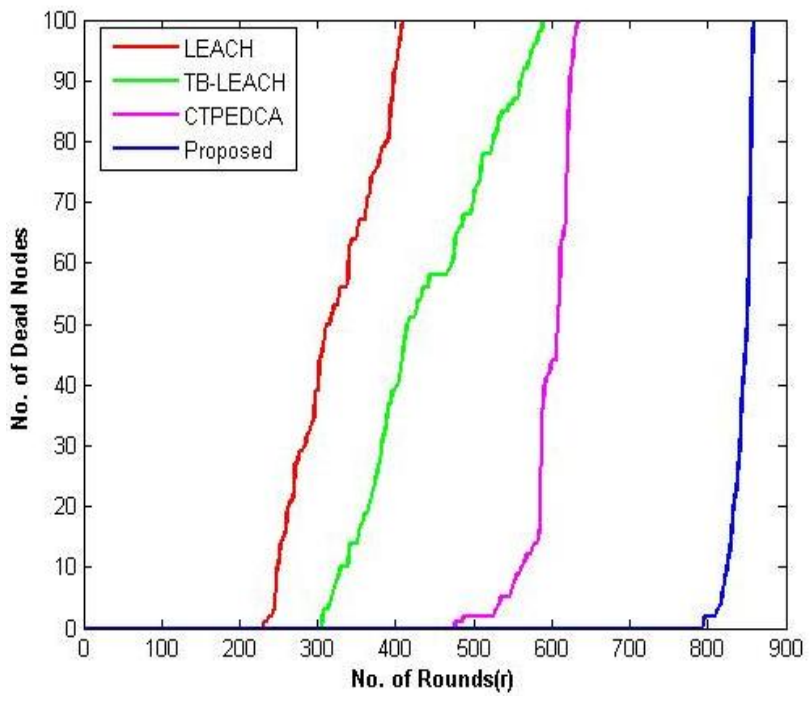

Fig. 6 Number of nodes dead over Simulation time when base station is located at $(\mathbf{5 0 , 2 7 5 )}$

Table 5. FND, HND and LND comparison at $(50,275)$

\begin{tabular}{|l|l|l|l|l|}
\hline $\begin{array}{l}\text { Base station } \\
\text { Loaction }\end{array}$ & Protocols & FND & HND & LND \\
\hline$(50,275)$ & LEACH & 230 & 310 & 408 \\
\cline { 2 - 5 } & TB-LEACH & 305 & 413 & 588 \\
\cline { 2 - 5 } & CTPEDCA & 475 & 607 & 633 \\
\cline { 2 - 5 } & Proposed & 794 & 850 & 858 \\
\hline
\end{tabular}

FND: It is clear from the table that first node dies later in the proposed protocol than the other three protocols. FND of the proposed protocol is approximately 3.4 times of LEACH, 2.6 times of TB-LEACH and 1.7 times of CTPEDCA.

Thus Stability period of proposed protocol is prolonger than LEACH, TB-LEACH and CTPEDCA protocols. CTPEDCA protocol is more stable than TB-LEACH and LEACH, while stability period of TB-LEACH is longer than LEACH protocol.

HND of proposed protocol is approximately 2.7 times of LEACH protocol, 2.1 times of TB-LEACH and 1.3 times CTPEDCA protocol. HND of CTPEDCA protocol is more than TB-LEACH and LEACH, while HND of TB-LEACH is more than LEACH protocol.

The proposed protocol has improved the network life time by about $110 \%$ as compared to $\mathrm{LEACH}$, about $46 \%$ as compared to TB-LEACH and about $35 \%$ as compared to CTPEDCA. The CTPEDCA and TB-LEACH protocols have improved the network life time as compare to LEACH protocol. The instability period of proposed protocol is smallest as compared to other three protocols.

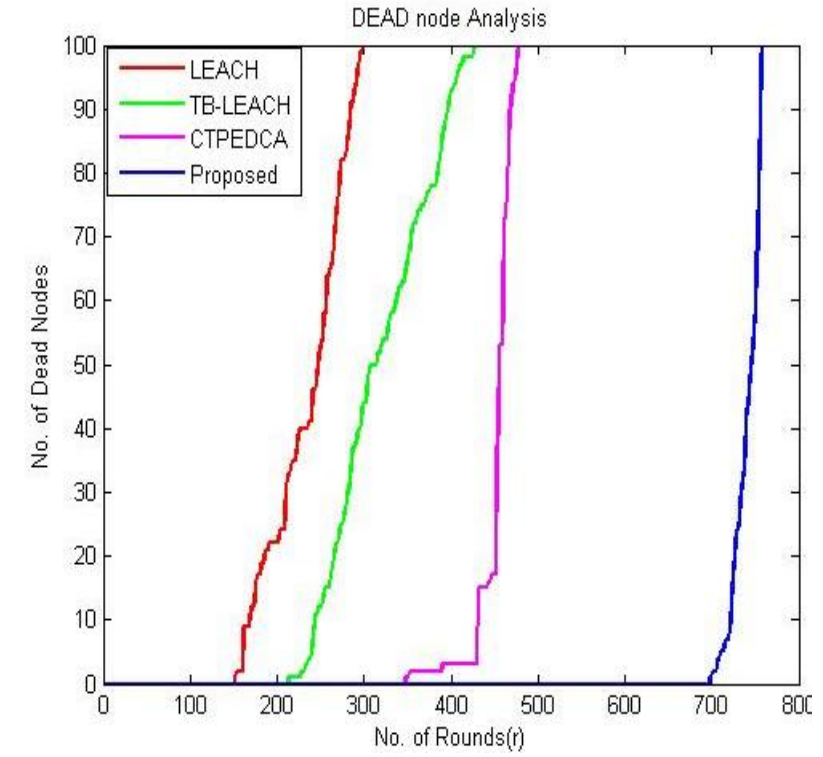

Fig. 7 Number of nodes dead over Simulation time when base station is located at $(\mathbf{5 0 , 3 0 0 )}$

Table 6. FND, HND and LND comparison at $(50,300)$

\begin{tabular}{|l|l|l|l|l|}
\hline $\begin{array}{l}\text { Base station } \\
\text { Loaction }\end{array}$ & Protocols & FND & HND & LND \\
\hline \multirow{3}{*}{$50,300)$} & LEACH & 151 & 247 & 298 \\
\cline { 2 - 5 } & TB-LEACH & 212 & 306 & 426 \\
\cline { 2 - 5 } & CTPEDCA & 347 & 455 & 477 \\
\cline { 2 - 5 } & Proposed & 697 & 745 & 757 \\
\hline
\end{tabular}

$F N D$ : It is clear from the table that first node dies later in the proposed protocol than the other three protocols. FND of the proposed protocol is approximately 3.4 times of LEACH, 2.6 times of TB-LEACH and 1.7 times of CTPEDCA.

Thus Stability period of proposed protocol is prolonger than LEACH, TB-LEACH and CTPEDCA protocols. CTPEDCA protocol is more stable than TB-LEACH and LEACH, while stability period of TB-LEACH is longer than LEACH protocol.

HND of proposed protocol is approximately 2.7 times of LEACH protocol, 2.1 times of TB-LEACH and 1.3 times CTPEDCA protocol. HND of CTPEDCA protocol is more than TB-LEACH and LEACH, while HND of TB-LEACH is more than LEACH protocol.

The proposed protocol has improved the network life time by about $110 \%$ as compared to LEACH, about $46 \%$ as compared to TB-LEACH and about $35 \%$ as compared to CTPEDCA. The CTPEDCA and TB-LEACH protocols have improved the network life time as compare to LEACH protocol. The instability period of proposed protocol is smallest as compared to other three protocols. 


\section{CONCLUSION}

To evaluate the performance of proposed protocol (hybrid TB-LEACH Energy Based Multihop Protocol) we have compared its performance with LEACH, TB-LEACH and CTPEDCA. The simulation results have shown that the proposed protocol has improved parameters FND and HND as compare to LEACH, TB-LEACH and CTPEDCA protocols when base station is at $(50,200)$. As the base station is moved more away the proposed protocol shows improvement in all the three parameters (FND, HND and LND) as compare to LEACH, TB-LEACH and CTPEDCA protocols. Thus proposed protocol has increased the stable region and network life time as compare to LEACH, TB-LEACH and CTPEDCA protocols. We concluded that proposed protocol performs better than other three protocols when base station is far distant. Performance of proposed protocol increases as the base station is moved away.

\section{REFERENCES}

[1] Surender Kumar, M.Prateek, N.J.Ahuja, and Bharat Bhushan, "DE-LEACH: Distance and Energy Aware LEACH", International Journal of Computer Applications, vol. 88, no.9, pp.36-42, February 2014.

[2] Fuad Bajaber and Irfan Awan, "Dynamic/Static Clustering Protocol for Wireless Sensor Network", Second UKSIM European Symposium on Computer Modeling and Simulation, IEEE, pp. 524-529, 2008.

[3] Zahra Beiranvand, Ahmad Patooghy, and Mahdi Fazeli, "I-LEACH: An efficient routing algorithm to improve performance \& to reduce energy consumption in Wireless Sensor Networks", 5th IEEE Conference on Information and Knowledge Technology (IKT), pp. 13-18, 2013.

[4] Erfan. Arbab, Vahe. Aghazarian, Alireza. Hedayati, and Nima. Ghazanfari Motlagh, "A LEACH-Based Clustering Algorithm for Optimizing Energy Consumption in Wireless Sensor Networks", 2nd International Conference on Computer Science and Information Technology (ICCSIT'2012), pp.147-150, April 2012.

[5] Muhammad Haneef, Zhou Wenxun, Zhongliang Deng, "MG-LEACH: Multi Group Based LEACH an Energy Efficient Routing Algorithm for Wireless Sensor Network", In Advanced Communication Technology (ICACT), 14th International Conference on IEEE, pp. 179-183, 2012
[6] Xiaowen Ma, Xiang Yu, "Improvement on LEACH Protocol of Wireless Sensor Network", Proceedings of the 2nd International Symposium on Computer, Communication, Control and Automation (ISCCCA-13), pp.338-341, 2012.

[7] Hui Gao, Yu Cheng, "A Relative Distance Based Clustering Scheme in Wireless Sensor Networks", Second International Conference on Intelligent Computation Technology and Automation, IEEE, pp. 426-429, 2009.

[8] Messai Mohamed-Lamine, "NEW CLUSTERING SCHEME FOR WIRELESS SENSOR NETWORKS" 8th International Workshop on Systems, Signal Processing and their Applications (WoSSPA), IEEE, pp. 487-491, 2013.

[9] Baiping $\mathrm{Li}$ and Xiaoqin Zhang, "Research and Improvement of LEACH Protocol for Wireless Sensor Network", International Conference on Information Engineering, pp. 48-54, 2012.

[10] Wei Wang, Bingwen Wang, Zhuo Liu, Lejiang Guo, and Wei Xiong, "A cluster-based and tree-based power efficient data collection and aggregation protocol for wireless sensor networks", Information Technology Journal,vol. 10, no. 3, pp. 557564, 2011.

[11] Hu"seyin O" zgür Tan and I'brahim Ko"rpeog`lu, "Power Efficient Data Gathering and Aggregation Wireless Sensor Networks", SIGMOD Record, vol. 32, no. 4, December 2003

[12] Tripti Sharma, Brijesh Kumar, and Geetam Singh Tomar, "Performance Comparision of LEACH, SEP and DEEC Protocol in Wireless Sensor Network," Proc. of the Intl. Conf. on Advances in Computer Science and Electronics Engineering, 2012.

[13] Hu Junping, Jin Yuhui and Dou Liang, "A Time-based Cluster-Head Selection Algorithm for LEACH", IEEE Symposium on Computers and Communications (ISCC), pp. 1172-1176, July 2008.

[14] H.Srikanth.Kamath, "Energy Efficient Routing Protocol for Wireless Sensor Networks", International Journal of Advanced Computer Research, vol. 3, issue10, June 2013.

[15] Gaganpreet Kaur, Sandeep Waraich, "Hybrid TBLEACH Energy Based Multihop Potocol", International Journal of International Journal of Computer Applications, volume 95, No.21, June 2014. 